\title{
An Existential Divisibility Lemma for Global Fields
}

\author{
Jeroen Demeyer* Jan Van Geel ${ }^{\dagger}$
}

2005-07-11

\begin{abstract}
We generalize the Existential Divisibility Lemma by Th. Pheidas ([Phe00] $)$ to all global fields $K$ of characteristic not 2, and for all sets of primes that are inert in a quadratic extension $L$ of $K$. We also remove the conditions in real and ramifying primes, which were present in Pheidas' version. As a Corollary, we recover the known fact that the set of integral elements at a prime in a global field is existentially definable.
\end{abstract}

Keywords: Diophantine definitions, Hilbert's Tenth Problem, Global fields.

AMS Subject Classification: 11 U05 (03D35 11E12 11R11).

\section{Introduction}

In [Phe00], Th. Pheidas proves what he calls an Existential Divisibility Lemma for $K=$ $\mathbb{F}_{q}(z)$ where $q$ is a prime congruent to $3 \bmod 4$ and for $K=\mathbb{Q}$. Let $R$ be the set of primes $p$ in $\mathbb{Z}$, respectively in $\mathbb{F}_{q}[z]$, such that -1 is not a square in the residue field of $p$. Let $v_{p}$ denote the normalized valuation associated to the prime $p$, and let $K^{*}=K \backslash\{0\}$. The Existential Divisibility Lemma states the existence of an existential formula $\phi(x, y)$ such that: if $\phi(x, y)$ holds for $x, y \in K^{*}$, then for all primes $p \in R$ for which $v_{p}(x)$ is odd it follows that $v_{p}\left(x y^{-2}\right)>0$.

Actually putting extra local conditions on the elements $x$ (conditions at the point at infinity in the function field case, at the real primes and the prime 2 in the case $K=\mathbb{Q}$ ) the truth of $\phi(x, y)$ for $x, y \in K^{*}$ is equivalent to the statement: for all primes $p \in R$ for which $v_{p}(x)$ is odd it follows that $v_{p}\left(x y^{-2}\right)>0$.

The formula $\phi(x, y)$ expresses the following: If $x$ has a pole at $p$ of odd multiplicity then $y$ has a pole at $p$ whose multiplicity is higher than half the multiplicity of $x$ at $p$. Also, if

\footnotetext{
*The first author is a Research Assistant of the Research Foundation - Flanders (FWO - Vlaanderen).

†Work partially supported by the European Community's Human Potential Programme under contract HPRN-CT-2002-00287.
} 
$y$ has a zero at $p$, and $x$ has odd order at $p$ then $x$ has a zero at $p$ of multiplicity larger than two times the multiplicity of $y$ at $p$.

This Existential Divisibility Lemma plays a role in strategies to obtain undecidability results for the existential theory of the field $K$. In [Phe91] Th. Pheidas proved that the existential theory of $\mathbb{F}_{q}(z)$ is undecidable. His proof worked for all odd primes $p$. C. Videla (Vid94) extended this result to rational global function fields of characteristic 2. A. Shlapentokh ([Shl96]) showed that the existential theory of global function fields of characteristic not 2 is undecidable and finally in her thesis ([Eis03]) K. Eisenträger completed the results by proving the same result for global function fields in characteristic 2. (This is essentially the (negative) solution of Hilbert's 10th problem for global function fields.) These undecidability results have the same starting point: The negative answer to Hilbert's Tenth problem, proven by Y. Matiyasevich, building on earlier work of M. Davis, H. Putnam and J. Robinson (cf. [Dav73] for a very good survey). This fact (that the existential theory of $\langle\mathbb{Z},+, \cdot\rangle$ is undecidable) implies that if a model of $\langle\mathbb{Z},+, \cdot\rangle$ can be defined in an existential way in any structure, the existential theory of that structure is undecidable.

One of the main open questions directly related to Hilbert's Tenth problem is whether or not the existential theory of $\mathbb{Q}$ (or more generally of any number field) is decidable or undecidable. In particular, an existential definition of $\langle\mathbb{Z},+, \cdot\rangle$ in $\langle\mathbb{Q},+, \cdot\rangle$ is not known. J. Robinson proved that every existential formula over $\langle\mathbb{Z},+, \cdot\rangle$ is equivalent to a formula over $\langle\mathbb{Q},+, \cdot\rangle$, however this formula contains several universal quantifiers.

In [Phe00, Th. Pheidas describes a program which might lead to a uniform way to give an existential definition of $\langle\mathbb{Z},+, \cdot\rangle$ in $\langle K,+, \cdot\rangle$, where $K$ is a global field. This program generates a series of "Possible Facts" (cf. [Phe00, sections 3 and 4]), two of which are related to Pheidas' Existential Divisibility Lemma and one of them is forced into the strategy because he uses the set of primes $p$ for which -1 is not a square in the residue field of $p$.

In view of this, Th. Pheidas and G. Cornelissen raised the question of the extent of which the Existential Divisibility Lemma holds: For which sets of primes does it hold? Why does the condition in the prime 2 occur? Can the Lemma be generalized to all global fields?

Together with K. Zahidi the second author worked out a more general version of the Existential Divisibility Lemma. Namely for $K=\mathbb{Q}$ and for a set of primes that are inert in a quadratic extension of $\mathbb{Q}$. This result was presented at the Oberwolfach meeting on Hilbert's 10th problem in January 2003 (cf. [VZ03]).

In this paper we generalize the Existential Divisibility Lemma to all global fields $K$ (of characteristic not 2), and for all sets of primes that are inert in a quadratic extension $L$ of $K$. We first prove the direct generalisation of Pheidas' Existential Divisibility Lemma, with conditions in the real primes and in the primes ramifying in $L$. In the last section we remove these conditions and prove:

Theorem (Existential Divisibility Lemma). Let $K$ be a global field of characteristic not 2 and $R(L / K)$ be the set of primes which are inert in a quadratic extension $L$ of $K$. Then there is an existential formula $\Omega(x, y)$ (depending on $K$ and $L$ ) with $x, y \in K^{*}$ 
which is equivalent to the formula

$$
\forall \mathfrak{r} \in R(L / K):\left(v_{\mathfrak{r}}(x) \text { odd } \rightarrow v_{\mathfrak{r}}\left(x y^{-2}\right)>0\right)
$$

If we take a finite number of quadratic extensions $L_{i} / K$, the statement still holds for the set of primes inert in at least one of these extensions:

Corollary. Let $K$ be a global field of characteristic not 2 and $L_{1}, \ldots, L_{k}$ quadratic extensions of $K$. Let $R$ be the set of primes which are inert in at least one extension $L_{i} / K$. Then there is an existential formula $\Theta(x, y)$ with $x, y \in K^{*}$ which is equivalent to the formula

$$
\forall \mathfrak{r} \in R:\left(v_{\mathfrak{r}}(x) \text { odd } \rightarrow v_{\mathfrak{r}}\left(x y^{-2}\right)>0\right)
$$

The language of these formulas is the language of rings $\mathcal{L}_{R}=\{0,1,+, \cdot\}$ with a symbol for $z$ if $K$ is a finite extension of $\mathbb{F}_{p}(z)$. Even though the formulas contain constants from $K$, this language suffices because these constants are algebraic over $\mathbb{Q}$ or $\mathbb{F}_{p}(z)$. Therefore, they can be defined (up to Galois actions) with a quantifier-free formula in the aforementioned language.

Using this version of the Existential Divisibility Lemma, G. Cornelissen and K. Zahidi prove ([CZ05]), conditionally on a conjecture about elliptic curves, that every existential formula over $\langle\mathbb{Z},+, \cdot\rangle$ is equivalent to a formula over $\langle\mathbb{Q},+, \cdot\rangle$ involving just one universal quantifier. Using an extra trick, they prove (again conditionally) that sentences over $\langle\mathbb{Q},+, \cdot\rangle$ of the form

$$
\left.\forall x \exists y_{1} \exists y_{2} \ldots \exists y_{n}: \varphi\left(x, y_{1}, \ldots, y_{n}\right) \quad \text { ( } \varphi \text { quantifier-free }\right)
$$

are undecidable.

Finally, we get the following known fact as a consequence of our Existential Divisibility Lemma:

Corollary (Integrality at a Prime). Let $K$ be a global field of characteristic not 2 and let $\mathfrak{p}$ be a non-archimedean prime in $K$. Then the set $\left\{z \in K \mid v_{\mathfrak{p}}(z) \geq 0\right\}$ is diophantine.

This Corollary can be seen as a version of the Existential Divisibility Lemma with a single prime (instead of infinitely many). Historically, this is how the Existential Divisibility Lemma came to be. It is due to different authors (in different cases, cf. the introduction of chapter 5 in [Eis03]), but the ideas go back to a couple of Lemmas in [Rob49] from 1949 by J. Robinson. She uses the Hasse-Minkowski Theorem for quadratic forms. R. Rumely gives a different proof ([Rum80]), using the Hasse norm principle.

\section{Preliminaries}

Our discussion of the Existential Divisibility Lemma relies on facts about norms and norm groups of quadratic extensions of local and global fields. We use the Hasse-Minkowski 
local-global principle and Hilbert's Reciprocity Law. In this section we give a survey of these facts, for more details and proofs we refer to the literature (e.g. ['M63]).

We start fixing terminology and notation. Throughout the paper $K$ will be a global field of characteristic not 2 , so it is either a number field or the function field of a curve over a finite field $\mathbb{F}_{q}$ with $q$ odd. $L$ will be a quadratic extension of $K$.

By $M_{K}$ we denote the set of all 'primes' $\mathfrak{p}$ of $K$. A prime $\mathfrak{p}$ can be non-archimedean or archimedean. The non-archimedean primes correspond (one to one) to (equivalence classes of) discrete valuations of $K$. In the function field case all primes are non-archimedean. In the number field case, there are in addition a finite number of archimedean primes, which correspond (one to one) to the different embeddings of $K$ in the complex numbers. Every element $\mathfrak{p} \in M_{K}$ gives rise to a normalized absolute value ||$_{\mathfrak{p}}$ on $K$, let $K_{\mathfrak{p}}$ denote the completion of $K$ with respect to ||$_{\mathfrak{p}}$.

If $\mathfrak{p}$ is a non-archimedean prime, then $K_{\mathfrak{p}}$ is the fraction field of a complete discrete valuation ring $\mathcal{O}_{\mathfrak{p}}$ and its maximal ideal is a principal ideal. A generator for this ideal is called a uniformizing element. We can choose such a uniformizing element in the base field $K$ and denote it with $\pi_{\mathfrak{p}}$. The quotient ring $\mathcal{O}_{\mathfrak{p}} /\left(\pi_{\mathfrak{p}}\right)$ is a finite field $\mathbb{F}_{\mathfrak{p}}$, the residue field of the prime $\mathfrak{p}$. The discrete valuation associated with a non-archimedean prime $\mathfrak{p}$ will be denoted with $v_{\mathfrak{p}}$ and we normalize it by $v_{\mathfrak{p}}\left(\pi_{\mathfrak{p}}\right)=1$.

All absolute values ||$_{\mathfrak{p}}$ extend to the quadratic extension $L / K$ in different ways, depending on the prime $\mathfrak{p}$. The possible extensions correspond to what is called the splitting behavior of the prime $\mathfrak{p}$ in $L$. For a quadratic extension we have the following cases (for number fields we distinguish between non-archimedean and archimedean primes, for function fields the cases 4 and 5 do not occur):

1. $\mathfrak{p}$ is a non-archimedean prime and $L \otimes_{K} K_{\mathfrak{p}} \cong K_{\mathfrak{p}} \times K_{\mathfrak{p}}$. In this case there are two primes $\mathfrak{P}_{1}, \mathfrak{P}_{2}$ in $M_{L}$ lying over $\mathfrak{p}, L_{\mathfrak{P}_{1}} \cong L_{\mathfrak{P}_{2}} \cong K_{\mathfrak{p}}$ and $\mathbb{F}_{\mathfrak{P}_{1}} \cong \mathbb{F}_{\mathfrak{P}_{2}} \cong \mathbb{F}_{\mathfrak{p}}$. We say that the prime $\mathfrak{p}$ is completely split in $L$.

2. $\mathfrak{r}$ is a non-archimedean prime and $L \otimes_{K} K_{\mathfrak{r}}$ is a quadratic field extension $L_{\mathfrak{R}}$ over $K_{\mathfrak{r}}$ and $\left[\mathbb{F}_{\mathfrak{R}}: \mathbb{F}_{\mathfrak{r}}\right]=2$. In this case there is only one prime $\mathfrak{R}$ in $L$ lying over $\mathfrak{r}$, the uniformizing element $\pi_{\mathfrak{r}}$ of $\mathcal{O}_{\mathfrak{r}}$ is also a uniformizing element of $\mathcal{O}_{\mathfrak{R}}$. The prime $\mathfrak{r}$ is said to be inert in $L$.

3. $\mathfrak{s}$ is a non-archimedean prime and $L \otimes_{K} K_{\mathfrak{s}}$ is a quadratic field extension $L_{\mathfrak{S}}$ over $K_{\mathfrak{s}}$ and $\left[\mathbb{F}_{\mathfrak{S}}: \mathbb{F}_{\mathfrak{s}}\right]=1$. Here we also have a unique prime $\mathfrak{S}$ in $L$ lying over $\mathfrak{s}$, but the uniformizing element $\pi_{\mathfrak{s}}$ of $\mathcal{O}_{\mathfrak{s}}$ is not a uniformizing element of $\mathcal{O}_{\mathfrak{S}}$. We have $\pi_{\mathfrak{S}}^{2}=w \pi_{\mathfrak{s}}$ with $w$ a unit in $\mathcal{O}_{\mathfrak{S}}$. The prime $\mathfrak{s}$ is said to ramify in $L$.

4. $\mathfrak{a}$ is an archimedean prime and $L \otimes K_{\mathfrak{a}} \cong K_{\mathfrak{a}} \times K_{\mathfrak{a}}$, the archimedean prime $\mathfrak{a}$ is said to split in $L$.

5. $\mathfrak{c}$ is an archimedean prime and $L \otimes_{K} K_{\mathfrak{c}}$ is quadratic extension $L_{\mathfrak{C}}$ of $K_{\mathfrak{c}}$. Note that this case can only occur if $K_{\mathfrak{c}} \cong \mathbb{R}$ and we have $L_{\mathfrak{C}} \cong \mathbb{C}$, the archimedean prime $\mathfrak{c}$ does not split in $L$. 
We can now partition the set of primes $M_{K}$ according to the splitting behavior in the quadratic extension $L$ :

$$
\begin{aligned}
P(L / K) & =\left\{\mathfrak{p} \in M_{K} \mid \mathfrak{p} \text { is a non-archimedean prime, completely split in } L\right\} \\
R(L / K) & =\left\{\mathfrak{r} \in M_{K} \mid \mathfrak{r} \text { is a non-archimedean prime, inert in } L\right\} \\
S(L / K) & =\left\{\mathfrak{s} \in M_{K} \mid \mathfrak{s} \text { is a non-archimedean prime, ramified in } L\right\} \\
A_{\mathrm{s}}(L / K) & =\left\{\mathfrak{a} \in M_{K} \mid \mathfrak{a} \text { is an archimedean prime, split in } L\right\} \\
A_{\mathrm{ns}}(L / K) & =\left\{\mathfrak{c} \in M_{K} \mid \mathfrak{c} \text { is an archimedean prime, not split in } L\right\}
\end{aligned}
$$

In the number field case we sometimes need to refer to all archimedean primes therefore we define $A(L / K)=A_{\mathrm{s}}(L / K) \cup A_{\mathrm{ns}}(L / K)$. In the function field case the sets $A(L / K)$, $A_{\mathrm{s}}(L / K)$ and $A_{\mathrm{ns}}(L / K)$ are empty.

The sets $S(L / K), A_{\mathrm{s}}(L / K)$ and $A_{\mathrm{ns}}(L / K)$ are finite. The sets $P(L / K)$ and $R(L / K)$ are not empty, Chebotarev's Density Theorem yields that these sets are infinite and both have density $\frac{1}{2}$. (Another consequence of Chebotarev's Theorem states that the set of primes $P(L / K)$ that split completely determines uniquely the (Galois) extension $L / K$.)

The strong approximation Theorem [Neu92, page 204] implies

Proposition 1. Let $T$ be a finite set of primes in $K$ such that $A(L / K) \subset T$. Let $a_{\mathfrak{t}} \in K$ for $\mathfrak{t} \in T$. Choose a prime $\mathfrak{p}_{0}$ not in $T$. Then for all $\varepsilon>0$ there exists an element $x \in K$ such that

$$
\left|x-a_{\mathfrak{t}}\right|_{\mathfrak{t}}<\varepsilon \text { for all } \mathfrak{t} \in T \text { and }|x|_{\mathfrak{q}} \leq 1 \text { for all } \mathfrak{q} \in M_{K} \backslash\left(T \cup\left\{\mathfrak{p}_{0}\right\}\right)
$$

As an immediate consequence of this one has

Corollary 2. Let $T, \mathfrak{p}_{0} \notin T$ and $a_{\mathfrak{t}} \in K$ be as in the preceding Proposition. Then there exists an element $a \in K$ such that

$$
\begin{aligned}
v_{\mathfrak{t}}(a) & =v_{\mathfrak{t}}\left(a_{\mathfrak{t}}\right) \text { for all } \mathfrak{t} \in T \backslash A(L / K) \\
\operatorname{sign}(a) & =\operatorname{sign}\left(a_{\mathfrak{t}}\right) \text { for all } \mathfrak{t} \text { with } K_{\mathfrak{t}} \cong \mathbb{R}
\end{aligned}
$$

and

$$
v_{\mathfrak{q}}(a) \geq 0 \text { for all } \mathfrak{q} \in M_{K} \backslash\left(T \cup\left\{\mathfrak{p}_{0}\right\}\right)
$$

Let $\sigma \in \operatorname{Gal}(L / K)$ be the non-trivial automorphism of $L$ fixing $K$. The norm and trace maps are defined as

$$
\mathrm{N}_{L / K}: L \rightarrow K: z \mapsto z \sigma(z) \quad \text { and } \quad \operatorname{Tr}_{L / K}: L \rightarrow K: z \mapsto z+\sigma(z)
$$

Let $L=K(\alpha)$ and choose the basis $\{1, \alpha\}$ for the 2-dimensional vector space $L$ over $K$. With respect to this basis, the norm becomes a quadratic form with coefficients in $K$ :

$$
N(x, y)=x^{2}+\operatorname{Tr}_{L / K}(\alpha) x y+\mathrm{N}_{L / K}(\alpha) y^{2}
$$

We call this the norm form, which we also denote by $N$. 
Facts. With notations as above, we have (cf. ['M63] and [Sch85]):

1. For all $\mathfrak{p} \in P(L / K)$ the norm form $N$ has a non-trivial zero over $K_{\mathfrak{p}}$. It follows that every element of $K_{\mathfrak{p}}$ is represented by $N$ (i.e., every elemeny is equal to $N(x, y)$ for certain $\left.x, y \in K_{\mathfrak{p}}\right)$.

2. For all $\mathfrak{r} \in R(L / K)$ the norm form $N$ has no non-trivial zero over $K_{\mathfrak{r}}$. The elements of $K_{\mathfrak{r}}$ represented by $N$ are exactly the norms of the extension $L_{\mathfrak{R}} / K_{\mathfrak{r}}$, where $\mathfrak{R}$ is the unique prime in $L$ lying over $\mathfrak{r}$. The norm map $\mathrm{N}_{L_{\mathfrak{R}} / K_{\mathfrak{r}}}$ is surjective on units $\mathcal{U}_{\mathfrak{r}}$ in $\mathcal{O}_{\mathfrak{r}}$ and the uniformizing element $\pi_{\mathfrak{r}}$ is not a norm. It follows that an element $x \in K_{\mathfrak{r}}$ is a norm if and only if its valuation $v_{\mathfrak{r}}(x)$ is even (we recall that $v_{\mathfrak{r}}$ is the discrete valuation associated to $\mathfrak{r}$ and normalized by $v_{\mathfrak{r}}\left(\pi_{\mathfrak{r}}\right)=1$ ).

3. For all $\mathfrak{s} \in S(L / K)$ the norm form $N$ has no non-trivial zero over $K_{\mathfrak{s}}$. Again the elements represented by $N$ are the norms of the extension $L_{\mathfrak{S}} / K_{\mathfrak{s}}$, where $\mathfrak{S}$ is the unique prime in $L$ lying over $\mathfrak{s}$. The image $\mathrm{N}_{L_{\mathfrak{S}} / K_{\mathfrak{s}}}\left(\mathcal{U}_{\mathfrak{S}}\right)$ of the group of units in $\mathcal{O}_{\mathfrak{S}}$ is a subgroup of index 2 in the group $\mathcal{U}_{\mathfrak{s}}$ of units in $\mathcal{O}_{\mathfrak{s}}$. It follows that $\mathrm{N}_{L_{\mathfrak{S}} / K_{\mathfrak{s}}}\left(L_{\mathfrak{S}}\right)$ is a subgroup of index 2 in $K_{\mathfrak{s}}$, since $\mathrm{N}_{L_{\mathfrak{S}} / K_{\mathfrak{s}}}\left(\pi_{\mathfrak{S}}\right)=u \pi_{\mathfrak{s}}$ for some unit $u$ in $\mathcal{O}_{\mathfrak{s}}$.

4. For primes $\mathfrak{a} \in A_{\mathrm{s}}(L / K)$ we have the same as completely split primes, namely that every element of $K_{\mathfrak{a}}$ is represented by $N$.

5. If $\mathfrak{c} \in A_{\mathrm{ns}}(L / K)$, the norm form $N$ has no non-trivial zero over $K_{\mathfrak{c}}$. The elements represented by $N$ are the norms of the extension $L_{\mathfrak{C}}=\mathbb{C}$ over $K_{\mathfrak{p}}=\mathbb{R}$. So these are the elements represented as sums of 2 squares in $\mathbb{R}$, which are exactly the positive elements in $\mathbb{R}$.

For inert primes $\mathfrak{r} \in R(L / K)$ we need slightly more than the fact that the norms in $K_{\mathfrak{r}}$ are the elements of even valuation.

Lemma 3. Let $\mathfrak{r} \in R(L / K)$ be an inert prime and $\mathfrak{R}$ the unique prime in L lying over r. Let $\alpha \in \mathcal{O}_{\mathfrak{R}}$ be such that $\mathcal{O}_{\mathfrak{R}} /\left(\pi_{\mathfrak{R}}\right)=\mathbb{F}_{\mathfrak{R}}=\mathbb{F}_{\mathfrak{r}}[\bar{\alpha}]=\left(\mathcal{O}_{\mathfrak{r}} /\left(\pi_{\mathfrak{r}}\right)\right)[\bar{\alpha}]$, with $\bar{\alpha}$ the reduction of $\alpha \bmod \Re$. Then

$$
v_{\mathfrak{r}}\left(\mathrm{N}_{L_{\mathfrak{R}} / K_{\mathfrak{r}}}(x+y \alpha)\right)=\min \left(v_{\mathfrak{r}}\left(x^{2}\right), v_{\mathfrak{r}}\left(y^{2}\right)\right)
$$

for $x, y \in K_{\mathfrak{r}}$.

Proof. Because $\mathfrak{r}$ is inert, we may replace $v_{\mathfrak{r}}$ with $v_{\mathfrak{R}}$ in the statement.

Note that $\sigma \in \operatorname{Gal}(L / K)$ extends to a generator of $\operatorname{Gal}\left(L_{\mathfrak{R}} / K_{\mathfrak{r}}\right)$, so

$$
v_{\mathfrak{R}}\left(\mathrm{N}_{L_{\mathfrak{R}} / K_{\mathfrak{r}}}(x+y \alpha)\right)=v_{\mathfrak{R}}((x+y \alpha)(x+y \sigma(\alpha)))=2 v_{\mathfrak{R}}(x+y \alpha)
$$

It remains to prove that $v_{\mathfrak{R}}(x+y \alpha)=\min \left(v_{\mathfrak{R}}(x), v_{\mathfrak{R}}(y)\right)$.

The hypotheses imply that $v_{\mathfrak{r}}(\alpha)=0$, since $\bar{\alpha}$ is non-zero in $\mathbb{F}_{\mathfrak{R}}$. If $v_{\mathfrak{r}}(x) \neq v_{\mathfrak{r}}(y)$, the properties of discrete valuations immediately give us $v_{\mathfrak{R}}(x+y \alpha)=\min \left(v_{\mathfrak{R}}(x), v_{\mathfrak{R}}(y)\right)$.

Now suppose $v_{\mathfrak{R}}(x)=v_{\mathfrak{R}}(y)=m$ for a certain integer $m$. Then we have $x=\pi_{\mathfrak{r}}^{m} x_{0}$ and $y=\pi_{\mathfrak{r}}^{m} y_{0}$, with $x_{0}$ and $y_{0}$ elements of $\mathcal{O}_{\mathfrak{r}}$ having valuation 0 . 
Since $\{1, \bar{\alpha}\}$ is a basis for $\mathbb{F}_{\mathfrak{R}}$ over $\mathbb{F}_{\mathfrak{r}}$, we have that $\overline{x_{0}+y_{0} \alpha}=\overline{x_{0}}+\overline{y_{0}} \bar{\alpha}$ is a non-zero element of $\mathbb{F}_{\mathfrak{R}}$, so $v_{\mathfrak{R}}\left(x_{0}+y_{0} \alpha\right)=0$. Hence $v_{\mathfrak{R}}(x+y \alpha)=v_{\mathfrak{R}}\left(\pi_{\mathfrak{r}}^{m}\left(x_{0}+y_{0} \alpha\right)\right)=m=$ $\min \left(v_{\mathfrak{R}}(x), v_{\mathfrak{R}}(y)\right)$.

Information on the global norm group $\mathrm{N}_{L / K}\left(L^{*}\right) \subset K^{*}$ can be obtained from Hilbert's Reciprocity Law as expressed by the exact sequence (cf. [Rei75] and [’M63])

$$
0 \rightarrow{ }_{2} \operatorname{Br}(K) \rightarrow \bigoplus_{\mathfrak{p} \in M_{K}}{ }_{2} \operatorname{Br}\left(K_{\mathfrak{p}}\right) \stackrel{\sum_{\text {inv }}}{\rightarrow} \mathbb{Z} / 2 \mathbb{Z} \rightarrow 0
$$

Here ${ }_{2} \operatorname{Br}(F)$ denotes the 2-component (the elements of order two) of the Brauer group of the field $F$. The elements of the Brauer group of $F$ are classes of central simple algebras over $F$ and the group law is induced by the tensor product of algebras. In the case of local and global fields (of characteristic not 2) the (non-trivial) elements of the 2-component of the Brauer group are given by quaternion division algebras, which in turn can be represented by symbols $(a, b)$ with $a, b$ non-zero elements of the field. The symbol $(a, b)$ corresponds to the quaternion algebra with basis $\{1, i, j, k\}$ satisfying $i^{2}=a, j^{2}=b, i j=-j i=k=a b$.

We will use the following stronger facts:

Proposition 4 ([Rei75, section 31]). Over a local field $K_{\mathfrak{p}}$ there exists a unique quaternion division algebra $\mathbb{H}_{\mathfrak{p}}$, so ${ }_{2} \operatorname{Br}\left(K_{\mathfrak{p}}\right) \cong \mathbb{Z} / 2 \mathbb{Z}$.

This element $\mathbb{H}_{\mathfrak{p}}$ splits over any quadratic extension $E$ of $K_{\mathfrak{p}}$, i.e., $\mathbb{H}_{\mathfrak{p}} \otimes_{K_{\mathfrak{p}}} E$ is a full matrix algebra over $K_{\mathfrak{p}}$ and is therefore trivial in $\operatorname{Br}\left(K_{\mathfrak{p}}\right)$.

In the exact sequence (1) presenting Hilbert's Reciprocity Law the maps $\operatorname{inv}_{\mathfrak{p}}:{ }_{2} \operatorname{Br}\left(K_{\mathfrak{p}}\right) \rightarrow$ $\mathbb{Z} / 2 \mathbb{Z}$ are defined by $\operatorname{inv}_{\mathfrak{p}}\left(\mathbb{H}_{\mathfrak{p}}\right)=1 \in \mathbb{Z} / 2 \mathbb{Z}$. Proposition 4 says that inv is an isomorphism.

Proposition 5 ([Rei75, Theorem 30.4]). Let $F$ be a field, $\operatorname{char}(F) \neq 2$. A quaternion division algebra $\mathbb{H}$ over $F$ is represented by a symbol of the form $(a, b)$ with $a \in F^{*} \backslash F^{* 2}$ if and only if $\mathbb{H} \otimes F(\sqrt{a})$ is trivial in ${ }_{2} \operatorname{Br}(F)$. If $a \in F^{*} \backslash F^{* 2}$ then a symbol $(a, b)$ is trivial in ${ }_{2} \operatorname{Br}(F)$ if and only if $b \in \mathrm{N}_{F(\sqrt{a}) / F}\left(F(\sqrt{a})^{*}\right)$.

The second part of this Proposition together with Hilbert's Reciprocity Law yield the Hasse norm Theorem for quadratic extensions $L / K$. This Theorem states that an element in $K$ is a norm of an element in a quadratic extension $L$ if and only if it is a norm locally everywhere. In order to formulate the Hasse-Minkowski Theorem, which is a generalization of this, we need some definitions first.

Definition 6. A quadratic form $Q$ over $K$ is a homogeneous polynomial of degree 2, with coefficients in $K$ :

$$
Q\left(x_{1}, \ldots, x_{n}\right)=\sum_{i, j=1}^{n} a_{i j} x_{i} x_{j} \quad\left(x_{1}, \ldots, x_{n} \in K\right)
$$

$Q$ is said to be isotropic if it has a non-trivial zero (i.e., a zero with not all variables zero). We say that $Q$ represents an element $y$ if $Q\left(x_{1}, \ldots, x_{n}\right)=y$ for a certain choice $x_{1}, \ldots, x_{n} \in K$. 
Theorem 7 (Hasse-Minkowski, [Sch85]). A quadratic form $Q$ over a global field $K$ is isotropic over $K$ if and only if is isotropic over $K_{\mathfrak{p}}$ for all $\mathfrak{p} \in M_{K}$.

An element $x$ in a global field $K$ is represented by a quadratic form $Q$ over $K$ if and only if $x$ is represented by $Q$ over $K_{\mathfrak{p}}$ for all $\mathfrak{p} \in M_{K}$.

Let ${ }_{2} \operatorname{Br}(L / K)$ be the kernel of the natural group morphism ${ }_{2} \operatorname{Br}(K) \rightarrow{ }_{2} \operatorname{Br}(L)$. Clearly for any element $\omega$ in this kernel we have $\operatorname{inv}_{\mathfrak{p}}(\omega) \equiv 0 \bmod 2$ for $\mathfrak{p} \in P(L / K) \cup A_{s}(L / K)$. Hilbert's Reciprocity Law together with the approximation Theorem allows us to give different parameterizations of the finite subgroups of ${ }_{2} \mathrm{Br}(L / K)$. We will use this in section 4 .

Let $Q$ be a finite set of primes satisfying

$$
S(L / K) \cup A_{\mathrm{ns}}(L / K) \subseteq Q \subseteq R(L / K) \cup S(L / K) \cup A_{\mathrm{ns}}(L / K)
$$

Denote with ${ }_{2} \mathrm{Br}^{Q}(L / K)$ the subgroup of ${ }_{2} \operatorname{Br}(L / K)$ defined by

$$
{ }_{2} \operatorname{Br}^{Q}(L / K)=\left\{\omega \in{ }_{2} \operatorname{Br}(L / K) \mid \operatorname{inv} \mathfrak{l}(\omega) \equiv 0 \bmod 2 \text { for all } \mathfrak{l} \in M_{K} \backslash Q\right\}
$$

Hilbert's Reciprocity Law gives a one-to-one correspondence between the sets ${ }_{2} \operatorname{Br}^{Q}(L / K)$ and $\left\{Q_{0} \subseteq Q \mid \# Q_{0}\right.$ even $\}$. This correspondence is given by

$$
\omega \mapsto Q_{\omega}=\left\{\mathfrak{q} \in M_{K} \mid \operatorname{inv}_{\mathfrak{q}} \omega \equiv 1 \bmod 2\right\}
$$

The latter is a subset of $Q$ by the definition of ${ }_{2} \mathrm{Br}^{Q}(L / K)$.

Let $T$ be a finite subset of primes in $R(L / K) \backslash Q$, and fix a $\Delta \in K^{*}$ for which $L=K(\sqrt{\Delta})$. Take an $\omega \in{ }_{2} \mathrm{Br}^{Q}(L / K)$, then $\omega$ is of the form $(\Delta, \lambda)$ with $\lambda \in K^{*}$. We claim that we may choose $\lambda$ such that $v_{\mathfrak{r}}(\lambda) \geq 0$ for all $\mathfrak{r} \in R(L / K)$ and $v_{\mathfrak{t}}(\lambda)=0$ for all $\mathfrak{t} \in T$. To see this, we note that for $\mathfrak{t} \in T$ we have $\operatorname{inv}_{\mathfrak{t}}(\omega) \equiv 0 \bmod 2$, so $\lambda$ is a norm of $L_{\mathfrak{T}} / K_{\mathfrak{t}}$ and $v_{\mathfrak{t}}(\lambda)$ is even. Now we apply the approximation Theorem (Corollary 2 ) with $\mathfrak{p}_{0} \in P(L / K)$ to find an element $c \in K^{*}$ such that

$$
\begin{array}{ll}
v_{\mathfrak{r}}(c) \geq-\frac{v_{\mathfrak{r}}(\lambda)}{2} & \text { for all } \mathfrak{r} \in R(L / K) \\
v_{\mathfrak{t}}(c)=-\frac{v_{\mathfrak{t}}(\lambda)}{2} & \text { for all } \mathfrak{t} \in T
\end{array}
$$

Since $\omega=(\Delta, \lambda)=\left(\Delta, c^{2} \lambda\right)$, we may replace $\lambda$ by $c^{2} \lambda$ to obtain the desired conditions.

By choosing one such $\lambda$ for every $\omega \in{ }_{2} \mathrm{Br}^{Q}(L / K)$, we obtain a set $\Lambda_{Q, T}(L / K)$ parametrizing the elements of ${ }_{2} \mathrm{Br}^{Q}(L / K)$, we have:

Lemma 8. Let $Q, T$ and $\Lambda_{Q, T}=\Lambda_{Q, T}(L / K)$ be as above.

There is a one-to-one correspondence between $\Lambda_{Q, T}$ and the subsets of $Q$ with an even number of elements, given by

$$
\lambda \mapsto Q_{\lambda}=\left\{\mathfrak{q} \in Q \mid \operatorname{inv}_{\mathfrak{q}}(\Delta, \lambda) \equiv 1 \bmod 2\right\}
$$

Conversely for every subset $Q_{0}$ of $Q$ with $\# Q_{0} \in 2 \mathbb{Z}$ there is a unique $\lambda \in \Lambda_{Q, T}$ determined by the fact that $\lambda$ is a norm of $L_{\mathfrak{Q}} / K_{\mathfrak{q}}$ if and only if $\mathfrak{q} \notin Q_{0}$, where $\mathfrak{Q} \in M_{L}$ and $\mathfrak{q}$ is the prime in $K$ lying under $\mathfrak{Q}$. 
Proof. Except for the last statement this follows from the contruction of the set $\Lambda_{Q, T}$. The last statement follows from the fact that $\operatorname{inv}_{\mathfrak{l}}(\Delta, \lambda) \equiv 0 \bmod 2$ for all $\mathfrak{l} \notin Q$ and that $\operatorname{inv}_{\mathfrak{q}}(\Delta, \lambda) \equiv 1 \bmod 2$ if and only if $\lambda$ is not a norm from $L_{\mathfrak{Q}} / K_{\mathfrak{q}}$.

\section{Existential Divisibility Lemma}

As before $K$ is a global field (of characteristic not 2) and $L$ is a quadratic extension of $K$. We fix an element $\Delta \in K^{*}$ such that $L=K(\sqrt{\Delta})$.

Main Lemma 9. There exists an existential formula $\phi(x, y)$ such that:

1. Let $x$ and $y$ be elements of $K^{*}$ for which $\phi(x, y)$ is true. If $\mathfrak{r}$ is any prime in $R(L / K)$ such that $v_{\mathfrak{r}}(x)$ is odd, then $v_{\mathfrak{r}}\left(x y^{-2}\right)>0$.

2. $\phi(x, y)$ is true for all elements $x$ and $y$ of $K^{*}$ satisfying the following conditions:

(a) There exists at least one $\mathfrak{r} \in R(L / K)$ for which $v_{\mathfrak{r}}(x)$ is odd.

(b) For every $\mathfrak{r} \in R(L / K)$ with $v_{\mathfrak{r}}(x)$ odd, we have $v_{\mathfrak{r}}\left(x y^{-2}\right) \geq 0$.

(c) For every $\mathfrak{c} \in A_{\mathrm{ns}}(L / K)$, the element $x$ is positive in $K_{\mathfrak{c}} \cong \mathbb{R}$.

(d) For every $\mathfrak{s} \in S(L / K)$, the element $x$ is a norm from $L_{\mathfrak{S}}$, where $\mathfrak{S}$ is the unique prime lying above $\mathfrak{s}$.

To prove this Main Lemma, we need to use Lemma 3 for all inert primes $\mathfrak{r} \in R(L / K)$. However in general it is not possible to find a primitive element $\alpha \in L$ such that $\mathbb{F}_{\mathfrak{R}}=\mathbb{F}_{\mathfrak{r}}[\bar{\alpha}]$ for all $\mathfrak{r} \in R(L / K)$. The following Lemma will remedy this obstacle:

Lemma 10. Let $K$ and $L$ be as above. There exist elements $\alpha_{0}, \alpha_{1} \in L^{*}$ such that for all $\mathfrak{r} \in R(L / K)$ either $\mathbb{F}_{\mathfrak{R}}=\mathbb{F}_{\mathfrak{r}}\left[\overline{\alpha_{0}}\right]$ or $\mathbb{F}_{\mathfrak{R}}=\mathbb{F}_{\mathfrak{r}}\left[\overline{\alpha_{1}}\right]$.

Proof. We start by setting $\alpha_{0}=\sqrt{\Delta}$. Consider a $\mathfrak{r} \in R(L / K)$ such that $v_{\mathfrak{r}}(\Delta)=0$ and (in the number field case) $\mathfrak{r}$ is not a 2 -adic prime (i.e., it is not lying above the prime 2 in Q). Since $L_{\mathfrak{R}}=K_{\mathfrak{r}}(\sqrt{\Delta})$ is a quadratic extension of $K_{\mathfrak{r}}$, it follows from the assumptions on $\mathfrak{r}$ that $\bar{\Delta}$ is not a square in $\mathbb{F}_{\mathfrak{r}}$. This is a consequence of Hensel's Lemma since for non-2-adic primes a square in the residue field lifts to a square in the completion. So for these primes $\mathfrak{r}$ we have $\mathbb{F}_{\mathfrak{R}}=\mathbb{F}_{\mathfrak{r}}(\overline{\sqrt{\Delta}})$.

The remaining primes are exactly the inert 2-adic primes or the inert primes $\mathfrak{r}$ for which $v_{\mathfrak{r}}(\Delta) \neq 0$. This set $T$ of inert primes is finite, say $T=\left\{\mathfrak{r}_{1}, \ldots, \mathfrak{r}_{n}\right\}$. For all $i=1, \ldots, n$, we let $\mathfrak{R}_{i}$ be the unique prime in $L$ lying over $\mathfrak{r}_{i}$.

Consider the semi-local ring $\mathcal{O}_{T}=\cap_{i=1}^{n}\left(\mathcal{O}_{\mathfrak{r}_{i}} \cap K\right)$ and its integral closure $\widetilde{\mathcal{O}_{T}}=\cap_{i=1}^{n}\left(\mathcal{O}_{\mathfrak{R}_{i}} \cap\right.$ $L)$. The canonical morphisms

$$
\mathcal{O}_{T} \hookrightarrow \mathcal{O}_{\mathfrak{r}_{i}} \text { and } \widetilde{\mathcal{O}_{T}} \hookrightarrow \mathcal{O}_{\mathfrak{R}_{i}}
$$

induce isomorphisms $\mathcal{O}_{T} /\left(\pi_{\mathfrak{r}_{i}}\right) \cong \mathcal{O}_{\mathfrak{r}_{i}} /\left(\pi_{\mathfrak{r}_{i}}\right)=\mathbb{F}_{\mathfrak{r}_{i}}$ and $\widetilde{\mathcal{O}_{T}} /\left(\pi_{\mathfrak{R}_{i}}\right) \cong \mathcal{O}_{\mathfrak{R}_{i}} /\left(\pi_{\mathfrak{R}_{i}}\right)=\mathbb{F}_{\mathfrak{R}_{i}}$. For all $i=1, \ldots, n$ the residue fields $\mathbb{F}_{\mathfrak{R}_{i}}$ are degree 2 extensions of $\mathbb{F}_{\mathfrak{r}_{i}}$, so $\mathbb{F}_{\mathfrak{R}_{i}}=\mathbb{F}_{\mathfrak{r}_{i}}\left[\overline{\beta_{i}}\right]$. By the Chinese Remainder Theorem there exists an element $\alpha_{1} \in \widetilde{\mathcal{O}_{T}}$ such that $\alpha_{1} \equiv$ $\overline{\beta_{i}} \bmod \left(\pi_{\mathfrak{R}_{i}}\right)$. This implies $\mathbb{F}_{\mathfrak{R}_{i}}=\mathbb{F}_{\mathfrak{r}_{i}}\left[\overline{\alpha_{1}}\right]$ for all $i=1, \ldots, n$. 
We can now prove the Main Lemma.

Proof. Take $\alpha_{0}, \alpha_{1}$ by Lemma 10. We define $\phi(x, y)$ as

$$
\phi(x, y) \leftrightarrow \phi_{0}(x, y) \wedge \phi_{1}(x, y)
$$

where $\phi_{i}(x, y)$ is the formula

$$
\phi_{i}(x, y) \leftrightarrow \exists a, b, c \in K: 1+x \mathrm{~N}\left(y^{-1}+\alpha_{i} c\right)=a^{2}-\Delta b^{2}
$$

or, written in another way

$$
\phi_{i}(x, y) \leftrightarrow \exists a, b, c \in K:\left(1+x y^{-2}\right)+x y^{-1} \operatorname{Tr}\left(\alpha_{i}\right) c+x \mathrm{~N}\left(\alpha_{i}\right) c^{2}-a^{2}+\Delta b^{2}=0
$$

We will prove part 11 of the Main Lemma. For the sake of contradiction, assume that $\phi(x, y)$ holds, but $v_{\mathfrak{r}}\left(x y^{-2}\right) \leq 0$ for some prime $\mathfrak{r} \in R(L / K)$ for which $v_{\mathfrak{r}}(x)$ is odd. Since $v_{\mathfrak{r}}\left(x y^{-2}\right)$ is odd, necessarily $v_{\mathfrak{r}}\left(x y^{-2}\right)<0$.

We have $\mathbb{F}_{\mathfrak{R}}=\mathbb{F}_{\mathfrak{r}}\left[\alpha_{i}\right]$, with $i$ either equal to 0 or 1 . Lemma 3 implies $v_{\mathfrak{r}}\left(\mathrm{N}\left(y^{-1}+\alpha_{i} c\right)\right)=$ $\min \left(v_{\mathfrak{r}}\left(y^{-2}\right), v_{\mathfrak{r}}\left(c^{2}\right)\right) \leq v_{\mathfrak{r}}\left(y^{-2}\right)$. It follows that

$$
v_{\mathfrak{r}}\left(x \mathrm{~N}\left(y^{-1}+\alpha_{i} c\right)\right) \leq v_{\mathfrak{r}}\left(x y^{-2}\right)<0
$$

Thus $v_{\mathfrak{r}}\left(1+x \mathrm{~N}\left(y^{-1}+\alpha_{i} c\right)\right)=v_{\mathfrak{r}}\left(x \mathrm{~N}\left(y^{-1}+\alpha_{i} c\right)\right)$, which is odd. But $\phi_{i}(x, y)$ states that $1+x \mathrm{~N}\left(y^{-1}+\alpha_{i} c\right)$ is equal to $a^{2}-\Delta b^{2}$. The latter however has even valuation in $\mathfrak{r}$ since it is a norm, so we found our contradiction.

To prove part 2 of the Main Lemma, we assume $x$ and $y$ satisfy all the given conditions.

We claim that $\phi_{i}(x, y)(i=0,1)$ will be true if the following quadratic form is isotropic:

$$
Q_{i}(a, b, c, d)=\left(1+x y^{-2}\right) d^{2}+x y^{-1} \operatorname{Tr}\left(\alpha_{i}\right) c d+x \mathrm{~N}\left(\alpha_{i}\right) c^{2}-a^{2}+\Delta b^{2}
$$

Indeed, if $Q_{i}$ is isotropic but $\phi_{i}(x, y)$ does not hold, then $Q_{i}$ must have a solution with $d$ equal to zero:

$$
x \mathrm{~N}\left(\alpha_{i}\right) c^{2}-a^{2}+\Delta b^{2}=0
$$

Now choose a prime $\mathfrak{r} \in R(L / K)$ for which $v_{\mathfrak{r}}(x)$ is odd. Then $v_{\mathfrak{r}}\left(x \mathrm{~N}\left(\alpha_{i}\right) c^{2}\right)$ will be odd, but $v_{\mathfrak{r}}\left(a^{2}-\Delta b^{2}\right)$ is even, which gives a contradiction.

It remains to prove that $Q_{i}(i=0,1)$ is isotropic in $K$, or by applying the Theorem of Hasse-Minkowski (cf. Theorem 7) that $Q_{i}$ is isotropic over every completion of $K$. We check this by considering all possible primes in $M_{K}$.

Case 1: $\mathfrak{a} \in A(L / K)$

$K_{\mathfrak{a}} \cong \mathbb{R}$ or $\mathbb{C}$. Set $d=0, c=\frac{1}{\sqrt{x}}$ and $a$ and $b$ such that $\alpha_{i}=a+b \sqrt{\Delta}$. It follows that $Q_{i}(a, b, c, d)=0$. 
Case 2: $\mathfrak{p} \in P(L / K)$

Take $\mathfrak{P}$ as one of the two primes in $M_{L}$ lying over $\mathfrak{p}$. Since $L=K(\sqrt{\Delta})$ and $L_{\mathfrak{P}}=K_{\mathfrak{p}}$ it follows that $\Delta$ is a square in $K_{\mathfrak{p}}$. This means that the form $\langle-1, \Delta\rangle$, which is a subform of $Q_{i}$, is isotropic in $K_{\mathfrak{p}}$. So also $Q_{i}$ is isotropic in $K_{\mathfrak{p}}$.

Case 3: $\mathfrak{r} \in R(L / K)$

Suppose $v_{\mathfrak{r}}(x)$ is even. We know that $v_{\mathfrak{r}}\left(\mathrm{N}\left(\alpha_{i}\right)\right)$ will also be even, so we can write $x \mathrm{~N}\left(\alpha_{i}\right)=$ $\pi_{\mathfrak{r}}^{2 m} u$ with $v_{\mathfrak{r}}(u)=0$. If we set $d=0$ and $c=\pi_{\mathfrak{r}}^{-m}$, then the first three terms of (2) will be equal to the unit $u$. Since every unit in $K_{\mathfrak{r}}$ is a norm of the extension $L_{\mathfrak{R}}$, there exist elements $a$ and $b$ in $L_{\mathfrak{R}}$ such that $u=\mathrm{N}(a+b \sqrt{\Delta})$. This proves that $Q_{i}$ is isotropic.

If $v_{\mathfrak{r}}(x)$ is odd, it is given that $v_{\mathfrak{r}}\left(x y^{-2}\right)>0$. This implies that $v_{\mathfrak{r}}\left(1+x y^{-2}\right)=0$, so $1+x y^{-2}$ is a unit. If we set $c=0$ and $d=1$, we can conclude as above that $Q_{i}$ is isotropic.

Case 4: $\mathfrak{s} \in S(L / K)$

Setting $d=0$ and $c=1$ in $Q_{i}$ yields the equation $x \mathrm{~N}_{L_{\mathfrak{S}} / K_{\mathfrak{S}}}\left(\alpha_{i}\right)=\mathrm{N}_{L_{\mathfrak{S}} / K_{\mathfrak{5}}}(a+b \sqrt{\Delta})$. If we write this as

$$
x=\mathrm{N}_{L_{\mathfrak{S}} / K_{\mathfrak{s}}}\left(\frac{a+b \sqrt{\Delta}}{\alpha_{i}}\right)
$$

we see that this will always have a solution, because by assumption $x$ is a norm from $L_{\mathfrak{S}}$ over $K_{\mathfrak{s}}$, and $a+b \sqrt{\Delta}$ represents all elements of $L_{\mathfrak{S}}=K_{\mathfrak{s}}(\sqrt{\Delta})$.

Remark. Main Lemma 9 applied to $K=\mathbb{Q}$ and $L=\mathbb{Q}(i)$ is a direct generalistation of the Existential Divisibility Lemma proven by Th. Pheidas in [Phe00]. In the ramified primes (only the prime 2 in that case), he formulates alternatives for condition $(2 \mathrm{~d}$ ) ( $x$ being a local norm). It is possible to formulate such alternatives in the general case. We did not work this out explicitly since in Theorem 14 all conditions in the ramified primes will be removed.

We also like to point out that condition (2a), the existence of an inert prime where $x$ has an odd value, is not necessary. Even without this condition one can show that if the quadratic form (2) is isotropic, there also is a zero $(a, b, c, d)$ with $d=1$. Since removing this condition would not substantially simplify the following section, we preferred to keep the formulation of the Lemma analogous to that of Th. Pheidas.

\section{Removing all extra conditions}

In this section we will prove a version of the Existential Divisibility Lemma without the local conditions (2a), (2c) and (2d). from Main Lemma 9. This way we will find a formula which is equivalent to For all primes $\mathfrak{r}$ in $R(L / K)$ for which $v_{\mathfrak{r}}(x)$ is odd, it holds that $v_{\mathfrak{r}}\left(x y^{-2}\right)>0$.

As an application of this, we give an existential definition of the set of elements of $K$ having non-negative valuation in a prime. 
Lemma 11. There exists a number $k$ and elements $u_{1}, \ldots, u_{k} \in K$ with $v_{\mathfrak{r}}\left(u_{i}\right) \geq 0$ for all $\mathfrak{r} \in R(L / K)$, such that the following holds:

Take any finite set $T \subset R(L / K)$ and consider the semi-local ring $\mathcal{O}_{T}=\cap_{\mathfrak{t} \in T}\left(\mathcal{O}_{\mathfrak{t}} \cap K\right)$. Every $x \in \mathcal{O}_{T}$ can be written as

$$
x=u_{1} x_{1}^{2}+u_{2} x_{2}^{2}+\cdots+u_{k} x_{k}^{2}
$$

with $x_{i} \in \mathcal{O}_{T} \backslash\{0\}$.

Proof. Here we need to give different arguments for $K$ a global function field and for $K$ a number field.

Let $K$ be a global function field of characteristic not 2. Let $L$ and $T$ be as in the statement of the Theorem and let $x \in \mathcal{O}_{T}$. Choose $y \in \mathcal{O}_{T}$ such that $y \neq \pm 1$ and $x+y \neq \pm 1$ (this is possible since $\mathcal{O}_{T}$ is infinite). Then

$$
x=\left(\frac{x+y+1}{2}\right)^{2}-\left(\frac{x+y-1}{2}\right)^{2}+\left(\frac{y-1}{2}\right)^{2}-\left(\frac{y+1}{2}\right)^{2}
$$

Since $\frac{1}{2} \in \mathcal{O}_{T}$ we see that every $x \in \mathcal{O}_{T}$ is equal to an alternating sum of four non-zero squares in $\mathcal{O}_{T}$. It follows that the Lemma holds in this case with $k=4$ and $u_{1}=u_{3}=1$, $u_{2}=u_{4}=-1$.

To prove the Lemma in the number field case we first note that every integer $x \in \mathbb{Z}$ is represented over $\mathbb{Z}$ by the quadratic form $x_{1}^{2}-x_{2}^{2}+x_{3}^{2}-x_{4}^{2}$. Namely if $x$ is odd choose $y \in \mathbb{Z}$ such that $x+4 y \neq \pm 1$ and $y \neq \pm 1$, then

$$
x=\left(\frac{x+4 y+1}{2}\right)^{2}-\left(\frac{x+4 y-1}{2}\right)^{2}+(y-1)^{2}-(y+1)^{2} .
$$

If $x$ is even choose $y \in \mathbb{Z}$ such that $y$ is odd, $x+y \neq \pm 1$ and $y \neq \pm 1$, then

$$
x=\left(\frac{x+y+1}{2}\right)^{2}-\left(\frac{x+y-1}{2}\right)^{2}+\left(\frac{y-1}{2}\right)^{2}-\left(\frac{y+1}{2}\right)^{2} .
$$

Note that in these representations of $x \in \mathbb{Z}$ all the coordinates are non-zero.

We know that the ring of integers $\mathcal{O}_{K}$ of $K$ is a finitely generated $\mathbb{Z}$-module, say

$$
\mathcal{O}_{K}=a_{1} \mathbb{Z}+a_{2} \mathbb{Z}+\cdots+a_{n} \mathbb{Z}
$$

Set $k=4 n$ and for the $u_{i}$ 's we take $a_{1},-a_{1}, a_{1},-a_{1}, a_{2},-a_{2}, a_{2},-a_{2} \ldots, a_{n},-a_{n}, a_{n},-a_{n}$. Since any integer is represented with non-zero coordinates by the form $x_{1}^{2}-x_{2}^{2}+x_{3}^{2}-x_{4}^{2}$ it follows that every element of $\mathcal{O}_{K}$ is represented with non-zero coordinates by the form $u_{1} x_{1}^{2}+u_{2} x_{2}^{2}+\cdots+u_{4 n} x_{4 n}^{2}$.

Now consider $\mathcal{O}_{T}$ with $T$ a finite subset of $R(L / K)$. Every $x$ in $\mathcal{O}_{T}$ can be written as $y / z$ with $y$ and $z$ in $\mathcal{O}_{K}$. And since $\mathcal{O}_{T}$ is a principal ideal domain we may assume that $v_{\mathfrak{t}}(z)=0$ for all $\mathfrak{t} \in T$. It follows from the above that $y z=u_{1} x_{1}^{2}+\cdots+u_{k} x_{k}^{2}$, with $x_{1}, \ldots, x_{k}$ non-zero elements of $\mathcal{O}_{K}$. Then

$$
x=\frac{y z}{z^{2}}=u_{1}\left(\frac{x_{1}}{z}\right)^{2}+\cdots+u_{k}\left(\frac{x_{k}}{z}\right)^{2}
$$

For all $i=1, \ldots, k$ and $\mathfrak{t} \in T$ we have $x_{i} / z \neq 0$ and $v_{\mathfrak{t}}\left(x_{i} / z\right)=v_{\mathfrak{t}}\left(x_{i}\right) \geq 0$, which means that $x_{i} / z \in \mathcal{O}_{T}$. 
Definition 12. Given any subset $\Lambda \subseteq K^{*}$, we define the support of $\Lambda$ to be

$$
\operatorname{supp}(\Lambda)=\left\{\left.\mathfrak{p} \in M_{K}|\exists \lambda \in \Lambda:| \lambda\right|_{\mathfrak{p}} \neq 1\right\}
$$

Note that the condition " $|\lambda|_{\mathfrak{p}} \neq 1$ " is equivalent to " $v_{\mathfrak{p}}(\lambda) \neq 0$ " if $\mathfrak{p}$ is non-archimedean. If $\Lambda$ is finite, then $\operatorname{supp}(\Lambda)$ will also be finite.

Choose four primes $\mathfrak{r}_{1}, \mathfrak{r}_{2}, \mathfrak{r}_{3}, \mathfrak{r}_{4}$ in $R(L / K)$ and define the following finite set of primes:

$$
Q=\left\{\mathfrak{r}_{1}, \mathfrak{r}_{2}, \mathfrak{r}_{3}, \mathfrak{r}_{4}\right\} \cup S(L / K) \cup A_{\mathrm{ns}}(L / K)
$$

Let $T$ be any finite subset of $R(L / K) \backslash Q$ and $\Lambda_{Q, T}$ the subset of $K^{*}$ parameterizing ${ }_{2} \operatorname{Br}^{Q}(L / K)$ as given in Lemma 8. By construction of $\Lambda_{Q, T}$ it holds that $\operatorname{supp}\left(\Lambda_{Q, T}\right) \cap T=$ $\emptyset$.

Lemma 13. Let $\Lambda=\Lambda_{Q, T}$ be the set as defined above. There exists an existential formula $\psi_{\Lambda}(x, y)$ such that:

1. Let $x$ and $y$ be elements of $K^{*}$ for which $\psi_{\Lambda}(x, y)$ is true. If $\mathfrak{r}$ is any prime in $R(L / K) \backslash \operatorname{supp}(\Lambda)$ such that $v_{\mathfrak{r}}(x)$ is odd, then $v_{\mathfrak{r}}\left(x y^{-2}\right)>0$.

2. Suppose $x$ and $y$ are elements of $K^{*}$ such that for every $\mathfrak{r} \in R(L / K)$ with $v_{\mathfrak{r}}(x)$ odd, it holds that $v_{\mathfrak{r}}\left(x y^{-2}\right)>0$. Then $\psi_{\Lambda}(x, y)$ is true.

Proof. To construct the formula $\psi_{\Lambda}(x, y)$, we use the $\phi(x, y)$ obtained in Main Lemma 9 , and the $u_{i}$ from Lemma 11 . We also set $u_{0}=1$. We define $\psi_{\Lambda}(x, y)$ as

$$
\begin{aligned}
& \exists y_{0}, y_{1}, \ldots, y_{k}, z_{0}, z_{1}, \ldots, z_{k} \in K \text { : } \\
& \left(y_{0} z_{0}=1\right) \wedge \ldots \wedge\left(y_{k} z_{k}=1\right) \wedge\left(\left(u_{0} z_{0}^{2}+\cdots+u_{k} z_{k}^{2}\right) y^{2}=1\right) \\
& \wedge\left(\bigvee_{\lambda \in \Lambda} \phi\left(\lambda x, y_{0}\right)\right) \wedge \ldots \wedge\left(\bigvee_{\lambda \in \Lambda} \phi\left(\lambda x, y_{k}\right)\right)
\end{aligned}
$$

Note that the first two lines are equivalent to

$$
\exists y_{0}, y_{1}, \ldots y_{k} \in K^{*}:\left(u_{0} y_{0}^{-2}+\cdots+u_{k} y_{k}^{-2}=y^{-2}\right)
$$

To prove part 1, assume that $\psi_{\Lambda}(x, y)$ holds. Take any prime $\mathfrak{r} \in R(L / K) \backslash \operatorname{supp}(\Lambda)$ for which $v_{\mathfrak{r}}(x)$ is odd. $\psi_{\Lambda}(x, y)$ implies that for all $i=1, \ldots, k, \phi\left(\lambda_{i} x, y_{i}\right)$ is true for a certain $\lambda_{i} \in \Lambda$. Because $\mathfrak{r}$ is outside of the support of $\Lambda$, we know that $v_{\mathfrak{r}}\left(\lambda_{i} x\right)=v_{\mathfrak{r}}(x)$ is odd. But now $\phi\left(\lambda_{i} x, y_{i}\right)$ implies that $v_{\mathfrak{r}}\left(\lambda_{i} x y_{i}^{-2}\right)>0$, and this valuation is equal to $v_{\mathfrak{r}}\left(x y_{i}^{-2}\right)$. Lemma 11 gave us $v_{\mathfrak{r}}\left(u_{i}\right) \geq 0$, so we also have $v_{\mathfrak{r}}\left(x u_{i} y_{i}^{-2}\right)>0$. We conclude the proof of part 1 by observing that

$$
v_{\mathfrak{r}}\left(x y^{-2}\right)=v_{\mathfrak{r}}\left(x\left(u_{0} y_{0}^{-2}+\cdots+u_{k} y_{k}^{-2}\right)\right) \geq \min _{i=0}^{k} v_{\mathfrak{r}}\left(x u_{i} y_{i}^{-2}\right)>0
$$

For the proof of part 2, suppose we have $x, y \in K^{*}$ such that for every $\mathfrak{r} \in R(L / K)$ with $v_{\mathfrak{r}}(x)$ odd, the inequality $v_{\mathfrak{r}}\left(x y^{-2}\right)>0$ holds.

We can use approximation to get a $y_{0}^{-1}$ for which the following finitely many conditions are satisfied: 
(I) $v_{\mathfrak{r}}\left(y_{0}^{-1}\right)>v_{\mathfrak{r}}\left(y^{-1}\right)$ for all $\mathfrak{r} \in R(L / K) \backslash\left\{\mathfrak{r}_{1}, \mathfrak{r}_{2}, \mathfrak{r}_{3}, \mathfrak{r}_{4}\right\}$ for which $v_{\mathfrak{r}}(x)$ is odd.

(II) $v_{\mathfrak{r}_{j}}\left(y_{0}^{-1}\right)>-v_{\mathfrak{r}_{j}}(x) / 2$ for $j=1,3$.

(III) $v_{\mathfrak{r}_{j}}\left(y_{0}^{-1}-y^{-1}\right)=-v_{\mathfrak{r}_{j}}\left(2 y^{-1}\right)+$ an even number greater than $\max \left\{-v_{\mathfrak{r}_{j}}(x), 2 v_{\mathfrak{r}_{j}}\left(2 y^{-1}\right)\right\}$, for $j=2,4$.

We define these sets of primes:

$$
\begin{aligned}
T_{0} & =\left\{\mathfrak{r}_{1}, \mathfrak{r}_{3}\right\} \cup\left\{\mathfrak{r} \in R(L / K) \backslash\left\{\mathfrak{r}_{1}, \mathfrak{r}_{2}, \mathfrak{r}_{3}, \mathfrak{r}_{4}\right\} \mid v_{\mathfrak{r}}(x) \text { is odd }\right\} \\
T_{1}=T_{2}=\cdots=T_{k} & =\left\{\mathfrak{r}_{2}, \mathfrak{r}_{4}\right\} \cup\left\{\mathfrak{r} \in R(L / K) \backslash\left\{\mathfrak{r}_{1}, \mathfrak{r}_{2}, \mathfrak{r}_{3}, \mathfrak{r}_{4}\right\} \mid v_{\mathfrak{r}}(x) \text { is odd }\right\}
\end{aligned}
$$

Claim. There exist $y_{0}, y_{1}, \ldots, y_{k} \in K^{*}$ such that $u_{0} y_{0}^{-2}+\cdots+u_{k} y_{k}^{-2}=y^{-2}$ and for all $0 \leq i \leq k$ we have $v_{\mathfrak{r}}\left(x y_{i}^{-2}\right)>0$ for all $\mathfrak{r} \in T_{i}$.

Proof of claim. We have already constructed $y_{0}$. The case $i=0$ follows easily from (II) and (II) above, together with the hypotheses of part 2 .

For every $\mathfrak{r} \in T_{1}$ we will prove that $v_{\mathfrak{r}}\left(y^{-2}-y_{0}^{-2}\right)$ is even, and $v_{\mathfrak{r}}\left(y^{-2}-y_{0}^{-2}\right) \geq-v_{\mathfrak{r}}(x)$. Set

$$
a=y^{-2}-y_{0}^{-2}=-\left(y_{0}^{-1}-y^{-1}\right)\left(\left(y_{0}^{-1}-y^{-1}\right)+2 y^{-1}\right)
$$

If $\mathfrak{r}$ is either $\mathfrak{r}_{2}$ or $\mathfrak{r}_{4}$, then from (III) it follows that $v_{\mathfrak{r}}\left(y_{0}^{-1}-y^{-1}\right)>v_{\mathfrak{r}}\left(2 y^{-1}\right)$, so

$$
v_{\mathfrak{r}}(a)=v_{\mathfrak{r}}\left(y_{0}^{-1}-y^{-1}\right)+v_{\mathfrak{r}}\left(2 y^{-1}\right)=\text { an even number greater than }-v_{\mathfrak{r}}(x)
$$

If $\mathfrak{r}$ is not $\mathfrak{r}_{2}$ nor $\mathfrak{r}_{4}$, we know that $v_{\mathfrak{r}}(x)$ is odd and the hypotheses say that $v_{\mathfrak{r}}\left(y^{-2}\right)>$ $-v_{\mathfrak{r}}(x)$. Then (I) implies that $v_{\mathfrak{r}}(a)=v_{\mathfrak{r}}\left(y^{-2}-y_{0}^{-2}\right)=v_{\mathfrak{r}}\left(y^{-2}\right)$ is even and $v_{\mathfrak{r}}(a)>-v_{\mathfrak{r}}(x)$.

Now we use approximation to find a $\mu \in K^{*}$ for which

$$
v_{\mathfrak{r}}(\mu)=-v_{\mathfrak{r}}(a) / 2 \quad \text { for all } \mathfrak{r} \in T_{1}
$$

This way $\mu^{2} a$ is in the ring $\mathcal{O}_{T_{1}}$, and by applying Lemma 11 for this ring, we write $\mu^{2} a$ as

$$
\mu^{2} a=u_{1} w_{1}^{2}+\cdots+u_{k} w_{k}^{2} .
$$

If we set $y_{i}^{-1}=w_{i} / \mu$, then $v_{\mathfrak{r}}\left(y_{i}^{-2}\right)=2 v_{\mathfrak{r}}\left(w_{i}\right)-2 v_{\mathfrak{r}}(\mu) \geq 0+v_{\mathfrak{r}}(a)>-v_{\mathfrak{r}}(x)$ for $\mathfrak{r} \in T_{1}=T_{i}$.

This concludes the proof of the claim.

Using this, we will show that $\bigvee_{\lambda \in \Lambda} \phi\left(\lambda x, y_{0}\right)$ is true. The argument proving that $\bigvee_{\lambda \in \Lambda} \phi\left(\lambda x, y_{i}\right)$ is true for $i=1, \ldots k$ is completely analogous but with the role of the pairs $\left\{\mathfrak{r}_{1}, \mathfrak{r}_{3}\right\}$ and $\left\{\mathfrak{r}_{2}, \mathfrak{r}_{4}\right\}$ interchanged.

We construct a subset $Q_{0}$ of $Q$ of even cardinality. We start by taking the primes in $\left\{\mathfrak{r}_{2}, \mathfrak{r}_{4}\right\} \cup S(L / K) \cup A_{\mathrm{ns}}(L / K)$ for which $x$ is not a local norm. We add $\mathfrak{r}_{1}$ to $Q_{0}$ if $x$ is a norm from $L_{\mathfrak{R}_{1}}$. If necessary, we add $\mathfrak{r}_{3}$ to make sure $Q_{0}$ has an even number of elements. If we take the $\lambda \in \Lambda$ such that $\lambda$ is a local norm everywhere, except for the primes in $Q_{0}$, then $\lambda x$ will have the following properties: 
- $\lambda x$ is a local norm for all primes in $S(L / K) \cup A_{\mathrm{ns}}(L / K)$.

- $v_{\mathfrak{r}_{1}}(\lambda x)$ is odd.

- $v_{\mathfrak{r}_{2}}(\lambda x)$ is even and $v_{\mathfrak{r}_{4}}(\lambda x)$ is even.

- $v_{\mathfrak{r}}(\lambda x) \equiv v_{\mathfrak{r}}(x) \bmod 2$ for all $\mathfrak{r} \in R(L / K) \backslash\left\{\mathfrak{r}_{1}, \mathfrak{r}_{2}, \mathfrak{r}_{3}, \mathfrak{r}_{4}\right\}$.

We will now prove that $\phi\left(\lambda x, y_{0}\right)$ is true for this $\lambda \in \Lambda$. The choice of $\lambda$ already implies conditions 2a, 2c and 2d for part 2 of Main Lemma 9.

In order to prove condition $2 \mathrm{~b}$, take any prime $\mathfrak{r} \in R(L / K)$ for which $v_{\mathfrak{r}}(\lambda x)$ is odd. We see that $\mathfrak{r}$ cannot be $\mathfrak{r}_{2}$ or $\mathfrak{r}_{4}$. If $\mathfrak{r}$ is not $\mathfrak{r}_{1}$ nor $\mathfrak{r}_{3}$, the fact that $v_{\mathfrak{r}}(\lambda x)$ is odd implies that $v_{\mathfrak{r}}(x)$ is odd. In any case we have $\mathfrak{r} \in T_{0}$. Hence $v_{\mathfrak{r}}\left(\lambda x y_{0}^{-2}\right) \geq v_{\mathfrak{r}}\left(x y_{0}^{-2}\right)>0$ by the preceding claim.

The previous Lemma is a form of the Existential Divisibility Lemma without conditions in the real primes and ramifying primes. However, in one direction, it does not work for primes in $\operatorname{supp}\left(\Lambda_{Q, T}\right)$. By applying the Lemma two times for well chosen sets $\Lambda_{Q, T}$ and $\Lambda_{Q^{\prime}, T^{\prime}}$, we can solve this problem and obtain the main Theorem.

Theorem 14. There is an existential formula $\Omega(x, y)$ which is equivalent to the formula

$$
\forall \mathfrak{r} \in R(L / K):\left(v_{\mathfrak{r}}(x) \text { odd } \rightarrow v_{\mathfrak{r}}\left(x y^{-2}\right)>0\right)
$$

Proof. Take eight different primes $\left\{\mathfrak{r}_{1}, \mathfrak{r}_{2}, \mathfrak{r}_{3}, \mathfrak{r}_{4}, \mathfrak{r}_{1}^{\prime}, \mathfrak{r}_{2}^{\prime}, \mathfrak{r}_{3}^{\prime}, \mathfrak{r}_{4}^{\prime}\right\}$ in $R(L / K)$, and define $Q$ as $\left\{\mathfrak{r}_{1}, \mathfrak{r}_{2}, \mathfrak{r}_{3}, \mathfrak{r}_{4}\right\} \cup S(L / K) \cup A_{\text {ns }}(L / K)$. Take $T=\left\{\mathfrak{r}_{1}^{\prime}, \mathfrak{r}_{2}^{\prime}, \mathfrak{r}_{3}^{\prime}, \mathfrak{r}_{4}^{\prime}\right\}$ and let $\Lambda=\Lambda_{Q, T}$ be the corresponding set parameterizing ${ }_{2} \mathrm{Br}^{Q}(L / K)$.

Let $Q^{\prime}=\left\{\mathfrak{r}_{1}^{\prime}, \mathfrak{r}_{2}^{\prime}, \mathfrak{r}_{3}^{\prime}, \mathfrak{r}_{4}^{\prime}\right\} \cup S(L / K) \cup A_{\text {ns }}(L / K)$ and take $T^{\prime}=\left\{\mathfrak{r}_{1}, \mathfrak{r}_{2}, \mathfrak{r}_{3}, \mathfrak{r}_{4}\right\} \cup\left(\operatorname{supp}\left(\Lambda_{Q, T}\right) \cap\right.$ $R(L / K))$. Since $T^{\prime} \cap Q^{\prime}=\emptyset$ by the choice of $T$, the parameterizing set $\Lambda^{\prime}=\Lambda_{Q^{\prime}, T^{\prime}}$ is defined. Note that the choice of $T^{\prime}$ now implies $R(L / K) \cap \operatorname{supp}\left(\Lambda_{Q, T}\right) \cap \operatorname{supp}\left(\Lambda_{Q^{\prime}, T^{\prime}}\right)=\emptyset$.

Now we define

$$
\Omega(x, y) \leftrightarrow \psi_{\Lambda}(x, y) \wedge \psi_{\Lambda^{\prime}}(x, y)
$$

Suppose $\Omega(x, y)$ is true. $\psi_{\Lambda}(x, y)$ says that $v_{\mathfrak{r}}(x)$ odd implies $v_{\mathfrak{r}}\left(x y^{-2}\right)>0$ for $\mathfrak{r} \in R(L / K) \backslash$ $\operatorname{supp}(\Lambda) . \psi_{\Lambda^{\prime}}(x, y)$ says the same thing for $\mathfrak{r} \in R(L / K) \backslash \operatorname{supp}\left(\Lambda^{\prime}\right)$. Because $R(L / K) \cap$ $\operatorname{supp}(\Lambda) \cap \operatorname{supp}\left(\Lambda^{\prime}\right)=\emptyset$, we have it for all $\mathfrak{r}$ in $R(L / K)$.

If (3) is satisfied, then we know by Lemma 13 that $\psi_{\Lambda}(x, y)$ and $\psi_{\Lambda^{\prime}}(x, y)$ are both true.

Corollary 15. Let $L_{1}, \ldots, L_{k}$ be a finite set of quadratic extensions of $K$. Let $R=$ $\bigcup_{i=1}^{k} R\left(L_{i} / K\right)$. There is an existential formula $\Theta(x, y)$ which is equivalent to the formula

$$
\forall \mathfrak{r} \in R:\left(v_{\mathfrak{r}}(x) \text { odd } \rightarrow v_{\mathfrak{r}}\left(x y^{-2}\right)>0\right)
$$


Proof. By Theorem 14 there exists an existential formula $\Omega_{L_{i} / K}(x, y)$ corresponding to the set of inert primes in the quadratic extension $L_{i}$. Define

$$
\Theta(x, y) \leftrightarrow \Omega_{L_{1} / K} \wedge \cdots \wedge \Omega_{L_{k} / K}(x, y)
$$

One verifies immediately that $\Theta(x, y)$ is the formula we are looking for.

As mentioned in the introduction, it also follows from our Existential Divisibility Lemma that the set of elements integral at a prime $\mathfrak{p}$ is a diophantine set:

Corollary 16. For every non-archimedean prime $\mathfrak{p} \in M_{K}$, the set $\left\{z \in K \mid v_{\mathfrak{p}}(z) \geq 0\right\}$ is diophantine.

Proof. Let $\mathfrak{p} \in M_{K}$ be a non-archimedean prime. Choose another non-archimedean prime $\mathfrak{q} \neq \mathfrak{p}$. Choose $L / K$ a quadratic extension such that $\mathfrak{p} \in R(L / K)$ and $\mathfrak{q} \in S(L / K)$. To see that such an extension exists, let $K_{\mathfrak{p}}\left(\alpha_{\mathfrak{p}}\right)$ be the unique quadratic unramified extension of $K_{\mathfrak{p}}$ and $K_{\mathfrak{q}}\left(\beta_{\mathfrak{q}}\right)$ a totally ramified extension of degree two of $K_{\mathfrak{q}}$. Let $X^{2}+a_{\mathfrak{p}, 1} X+a_{\mathfrak{p}, 2}$ be the minimal polynomial of $\alpha_{\mathfrak{p}}$ over $K_{\mathfrak{p}}$ and $X^{2}+b_{\mathfrak{q}, 1} X+b_{\mathfrak{q}, 2}$ be the minimal polynomial of $\beta_{\mathfrak{q}}$ over $K_{\mathfrak{q}}$. Then Lemma (33.8) in [Rei75] states that if $c_{1}$ and $c_{2}$ are taken sufficiently close to $a_{\mathfrak{p}, 1}$ and $b_{\mathfrak{q}, 1}$, respectively $a_{\mathfrak{p}, 2}$ and $b_{\mathfrak{q}, 2}$, the polynomial $f(X)=X^{2}+c_{1} X+c_{2}$ is separable and irreducible over $K$ having a root in $K_{\mathfrak{p}}$ and in $K_{\mathfrak{q}}$. It follows that $K(\gamma)$, with $f(\gamma)=0$, is a quadratic extension of $K$ with the desired properties.

Let $\omega \in{ }_{2} \operatorname{Br}(K)$ such that $\operatorname{inv}_{\mathfrak{p}} \omega \equiv 1 \bmod 2, \operatorname{inv}_{\mathfrak{q}} \omega \equiv 1 \bmod 2$ and $\operatorname{inv}_{\mathfrak{r}} \omega \equiv 0 \bmod 2$ for all primes $\mathfrak{r} \neq \mathfrak{p}, \mathfrak{q}$. (Such an element $\omega$ exists by Hilbert's Reciprocity Law). By construction $L$ is a splitting field of $\omega$. So $\omega=(\gamma, x)$ with $L=K(\gamma)$. It follows from the choice of $L$ and $\omega$ that $v_{\mathfrak{p}}(x)$ is odd and $v_{\mathfrak{r}}(x)$ is even for all $\mathfrak{r} \in R(L / K) \backslash\{\mathfrak{p}\}$. After multiplying $x$ with a suitable square, we may assume that $v_{\mathfrak{p}}(x)=1$. Now apply Theorem 14 with this fixed $x$. Then $\Omega(x, y)$ is equivalent to " $v_{\mathfrak{p}}\left(x y^{-2}\right)>0$ ", or " $v_{\mathfrak{p}}\left(y^{-1}\right)>$ $-v_{\mathfrak{p}}(x) / 2$ ". If we set $y=z^{-1}$ and use the fact that $v_{\mathfrak{p}}(x)=1$, we find that $\Omega\left(x, z^{-1}\right)$ is equivalent to " $v_{\mathfrak{p}}(z) \geq 0$ ".

\section{References}

[CZ05] Gunther Cornelissen and Karim Zahidi, Complexity of undecidable formulce in the rationals and inertial Zsigmondy theorems for elliptic curves, Preprint, 2005.

[Dav73] Martin Davis, Hilbert's tenth problem is unsolvable, Amer. Math. Monthly 80 (1973), 233-269.

[Eis03] Kirsten Eisenträger, Hilbert's tenth problem and arithmetic geometry, Ph.D. thesis, University of California at Berkeley, 2003.

[Neu92] Jürgen Neukirch, Algebraische Zahlentheorie, Springer, 1992.

[O’M63] Timothy O'Meara, Introduction to quadratic forms, Springer, 1963.

[Phe91] Thanases Pheidas, Hilbert's tenth problem for rational function fields over finite fields, Invent. Math. 103 (1991), 1-8. 
[Phe00] - An effort to prove that the existential theory of $\mathbb{Q}$ is undecidable, Hilbert's Tenth Problem: Relations with Arithmetic and Algebraic Geometry (Ghent, 1999), Contemp. Math., vol. 270, 2000, pp. 237-252.

[Rei75] Irving Reiner, Maximal orders, London Academic Press, 1975.

[Rob49] Julia Robinson, Definability and decision problem in arithmetic, J. Symb. Log. 14 (1949), 98-114.

[Rum80] Robert Rumely, Undecidability and definability for the theory of global fields, Trans. Amer. Math. Soc. 262 (1980), 195-217.

[Sch85] Winfried Scharlau, Quadratic and hermitian forms, Grundlehren Math. Wiss., no. 270, Springer Berlin, 1985.

[Shl96] Alexandra Shlapentokh, Diophantine undecidability over algebraic function fields over finite fields of constants, J. Number Theory 58 (1996), 317-342.

[Vid94] Carlos Videla, Hilbert's tenth problem for rational function fields in characteristic 2, Proc. Amer. Math. Soc. 120 (1994), 249-253.

[VZ03] Jan Van Geel and Karim Zahidi, Quadratic forms and divisibility, MiniWorkshop: Hilbert's 10th problem, Mazur's Conjecture and Divisibility Sequences (Oberwolfach, January 19-25), 2003. 